\title{
Characterization of the Manufacturing Processes to Grow Triple-Junction Solar Cells
}

\author{
N. A. Kalyuzhnyy, ${ }^{1}$ V. V. Evstropov, ${ }^{1}$ V. M. Lantratov, ${ }^{1}$ S. A. Mintairov, ${ }^{1}$ M. A. Mintairov, ${ }^{1}$ \\ A. S. Gudovskikh, ${ }^{1,2}$ A. Luque, ${ }^{1,3}$ and V. M. Andreev ${ }^{1}$ \\ ${ }^{1}$ Ioffe Physical-Technical Institute of the Russian Academy of Sciences, Polytechnicheskaya Street 26, \\ Saint Petersburg 194021, Russia \\ ${ }^{2}$ Saint-Petersburg Academic University, Nanotechnology Research and Education Centre RAS, Hlopina Street 8/3, \\ Staint Petersburg 194021, Russia \\ ${ }^{3}$ Technical University of Madrid, Spain
}

Correspondence should be addressed to N. A. Kalyuzhnyy; nickk@mail.ioffe.ru and V. M. Andreev; vmandreev@mail.ioffe.ru

Received 12 December 2013; Revised 31 March 2014; Accepted 5 April 2014; Published 5 May 2014

Academic Editor: Niyaz Mohammad Mahmoodi

Copyright (c) 2014 N. A. Kalyuzhnyy et al. This is an open access article distributed under the Creative Commons Attribution License, which permits unrestricted use, distribution, and reproduction in any medium, provided the original work is properly cited.

\begin{abstract}
A number of important but little-investigated problems connected with III-V/Ge heterostructure in the GaInP/GaInAs/Ge multijunction solar cells grown by MOVPE are considered in the paper. The opportunity for successfully applying the combination of reflectance and reflectance anisotropy spectroscopy in situ methods for investigating III-V structure growth on a Ge substrate has been demonstrated. Photovoltaic properties of the III-V/Ge narrow-band subcell of the triple-junction solar cells have been investigated. It has been shown that there are excess currents in the Ge photovoltaic p-n junctions, and they have the tunneling or thermotunneling character. The values of the diode parameters for these current flow mechanisms have been determined. The potential barrier at the III-V/Ge interface was determined and the origin of this barrier formation during MOVPE heterogrowth was suggested.
\end{abstract}

\section{Introduction}

Multijunction (MJ) solar cells (SCs) grown by metal-organic vapor phase epitaxy (MOVPE) demonstrate the highest efficiency being one of the most successful trends of photovoltaic development [1-4]. Stacking several junctions with different band gaps allows reducing significantly the fundamental losses due to charge carrier thermalization. Triple-junction cells based on the $\mathrm{Ga}_{0.52} \mathrm{In}_{0.48} \mathrm{P} / \mathrm{Ga}_{0.99} \mathrm{In}_{0.01}$ As/Ge structure are currently the main part of the mass production of high efficiency multijunction solar cells. Although the material combination in terms of band gap does not give the maximum theoretical efficiency for triple-junction cells, the fact that they are lattice matched allows producing such monolithic heterostructure solar cells with a perfect crystalline quality.

The MJ SCs are widely used both in space and in terrestrial concentrator photovoltaic systems due to high efficiency under concentrated sunlight and high radiation resistance.

In the topological sense, the MJ SC structure is a series connection of single-junction subcells. Each subcell includes not only the photoactive $\mathrm{p}-\mathrm{n}$ junction but also the functional heterolayers (e.g., window, back surface field (BSF), etc.). Moreover the subcells are connected by tunnel junctions with additional heterointerfaces. As a result, a MJ SC has a quite complicated structure (see Figure 1), consisting of many layers with different types of interfaces including chemically dissimilar III-V/Ge interface (the GaInP/Ge heterostructure is used as the bottom subcell of the triple-junction GaInP/GaInAs/Ge SC). There are a number of important but little-investigated problems connected with this interface. First of all the electrical properties of the III-V/IV interface have not been enough studied so far, especially taking into account the diffusion processes, which take place during the Ge p-n junction formation during the MOVPE growth of 


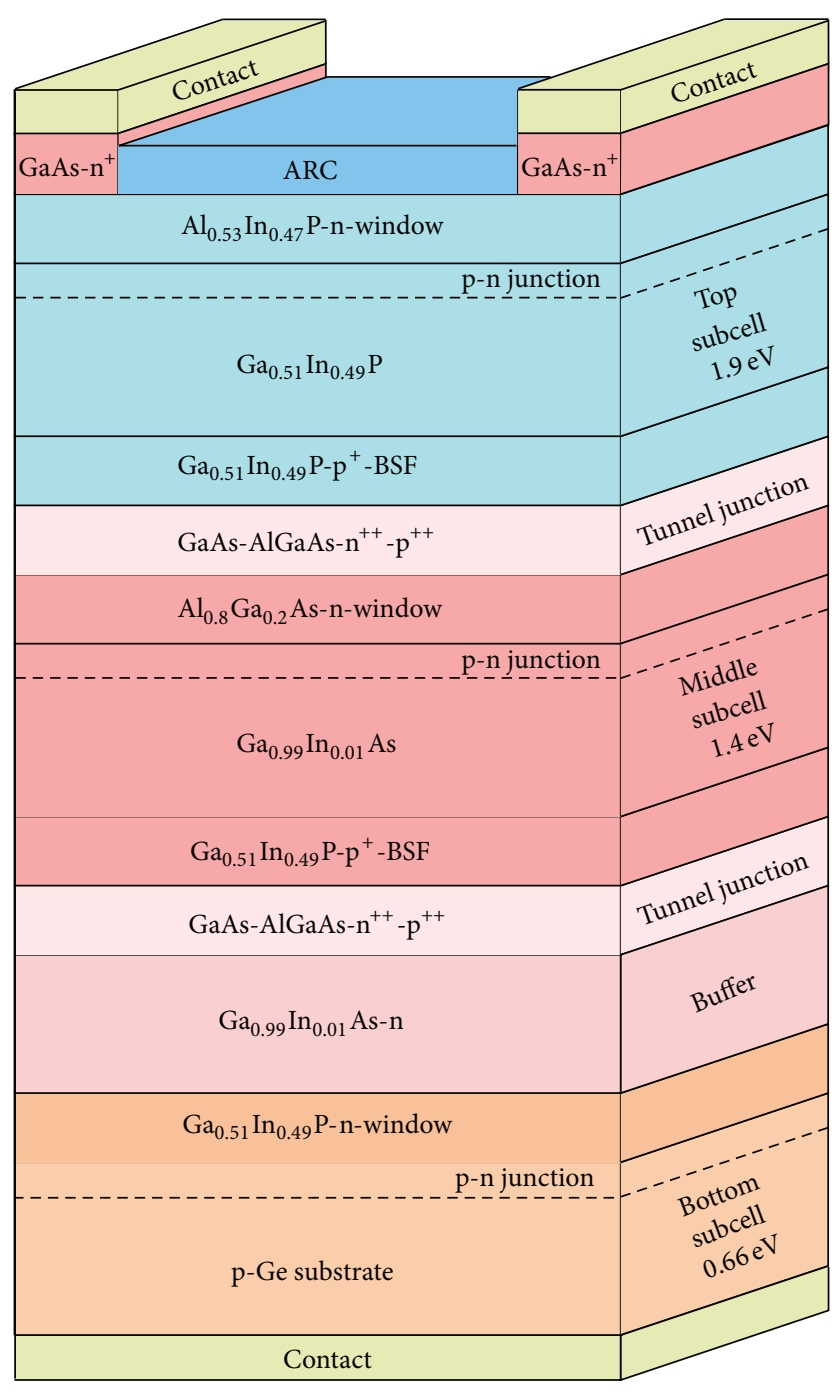

Figure 1: Schematic structure of a GaInP/GaInAs/Ge triple-junction solar cell.

III-V semiconductors on the Ge wafer. The interface phenomena may affect the charge carrier transport in the Ge subcell as well as in the whole multijunction solar cell.

In this paper a review of a number of results devoted to III-V/Ge heterostructure formed during MOVPE is presented. Also new approaches to control the epitaxial heterogrowth of III-V semiconductors on the germanium wafer during the MOVPE growth are shown.

\section{Materials and Methods}

The growth experiments have been performed in a horizontal low pressure R\&D MOVPE reactor. Hydrogen was used as a carrier gas. The following metalorganic compounds were used as the third group element sources: trimethylgallium (TMGa), trimethylaluminum (TMAl), and trimethylindium (TMIn). Arsine $\left(\mathrm{AsH}_{3}\right)$ and phosphine $\left(\mathrm{PH}_{3}\right)$ were used as the fifth group element sources. Doping precursors for investigated epitaxial layers were disilane $\left(\mathrm{SiH}_{4}\right)$ and diethylzinc
(DEZn). The experimental layers for in situ investigations as well as photovoltaic structures were grown on 2 -inch in diameter substrates.

The MOVPE installation is equipped with a UV-transparent quartz window for optical in situ measurements. A commercially available spectrometer has been used for performing reflectance $(\mathrm{R})$ as well as reflectance anisotropy (RA) signals on rotating samples.

According to Aspnes et al. [5], the reflectance anisotropy for cubic crystals is the difference in reflectance between the two surface symmetry axes along [110] and [-110] directions. For the (100) surface, the RA signal is defined by

$$
\frac{\Delta R}{R}=2 \frac{R_{110}-R_{-110}}{R_{110}+R_{-110}},
$$

where $R_{110}$ and $R_{-110}$ are the complex reflectance for the polarizations parallel to the [110] and [-110] directions.

The difference in the reflection coefficients for light polarized along the main crystalline axes results mainly from the surface anisotropy [6] (taking into account the bulk symmetry of cubic crystals). This anisotropy is characterized by the spatial configuration of the dimmers formed due to surface dangling bonds. Reflectance anisotropy spectroscopy (RAS) allows picking out weak signals of the surface optical transitions using polarization properties.

Due to sample rotation in MOVPE, the RA signal measurement is more complicated especially when it is accompanied by some degree of wobble [7]. Direct measurements during MOVPE growth processes give the absolute value of the RA signal that should be taken into account in analyzing experimental results.

The in situ measurements are an important part in investigating the semiconductor epitaxial structures and creating different semiconductor devices by the epitaxial method [8]. Investigations by RAS proved to be a useful instrument for determining a number of key parameters of epitaxial layers, semiconductor surfaces, and interfaces [9-12]. The RAS is applied effectively for comprehensive investigation of different semiconductor devices grown by the MOVPE technique, such as heterojunction bipolar transistors [7], laser structures [13], and vertical cavity surface-emitting lasers [14]. However, there is the quite small number of papers on RAS application for investigating the multijunction solar cell.

The approach for studying and controlling parameters of the MJ SC structures during MOVPE growth has been developed [15-17]. There are a number of factors decreasing the surface isotropy, such as surface reconstruction, morphology, atomic ordering, and surface electric field. The RA signal reflects contribution of all these factors. Comparison of the spectra of experimental samples with, for example, similar reconstruction allows obtaining a contribution of ordering and/or doping to their surface anisotropy. It has been demonstrated that the impurity concentration in the GaAs, GaInP, and $\mathrm{Ga}_{0.99} \mathrm{In}_{0.01}$ As layers grown on both $\mathrm{Ge}$ and GaAs substrates may be determined by the RA signal due to the linear electrooptical effect caused by the electric field of the near-surface space charge region $[15,16]$. It has been shown that $\mathrm{CuPt}_{\mathrm{B}}$-type ordering effect on the group 


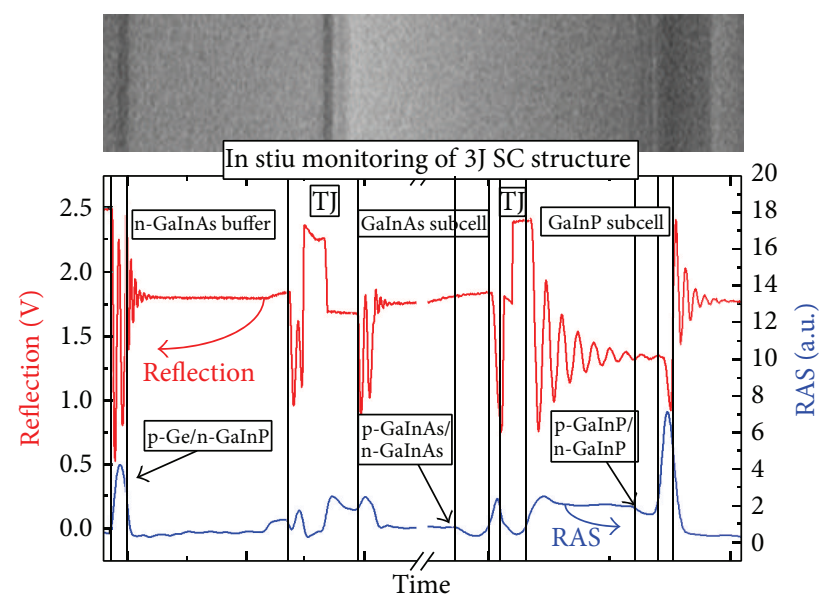

FIGURE 2: Approach of the in situ control of MJ SC parameters (layer thicknesses, solid solution composition, doping levels and homointerface detection, heterointerface quality, etc.) based on simultaneous measurements of the time dependencies of short-wavelength (on $3.8 \mathrm{eV}$ ) RA and long-wavelength (on $2.1 \mathrm{eV}$ ) reflection signals (lower part) and SEM image of cross-section of GaInP/GaInAs/Ge MJ SC.

III sublattice of GaInP [18], which results in narrowing the semiconductor band gap, can be determined by RAS at growth temperature of GaInP on both $\mathrm{Ge}$ and GaAs substrates [16].

A general method for the in situ control of MJ SC parameters (layer thicknesses, solid solution composition, doping levels, heterointerface quality, etc.) based on these studies has been proposed (Figure 2). The method is a combination of reflectance with the RAS, and it is based on simultaneously measuring the time dependence of the long-wavelength reflection signal and the short-wavelength anisotropic reflection signal $[16,19]$. On the one hand the long-wavelength (on $2.1 \mathrm{eV}$ ) measurements characterized by the larger absorption depth allow obtaining the quite large number of Fabry-Perot oscillations on the reflection, which is useful for composition, growth rate, and thickness of a material determination. On the other hand there is direct dependence of the $\triangle$ RAS (difference between signals for doped and undoped layers $\Delta \mathrm{RAS}=\Delta R / R_{\text {doped }}-\Delta R / R_{\text {undoped }}$ ) on the logarithm of the free charge carrier concentration for the short-wavelength range, so $3.8 \mathrm{eV}$ RA signal measurements allow controlling the doping level and observing homointerfaces. Also it is used for determining the quality of interfaces.

There is a wide range of ex situ techniques used for III-V interface characterization. The structural properties of interfaces can be studied by XRD [21]. The values of the recombination rate at interfaces can be measured using time resolved photoluminescence [22]. The values of the valence band offset $\left(\Delta E_{V}\right)$ and the interface charge density at interfaces can be obtained by CV profiling [23] among other methods.
However, there are a number of methods which are helpful but not often used for this purpose. First of all they are the above mentioned optical in situ methods, which allow estimating the heterogrowth quality. As an ex situ method, the admittance spectroscopy is helpful enough for studying the interface properties [24]. In general, capture and emission of charge carriers at and from interface states lead to variation of charge at the interface and, therefore, may contribute to the structure capacitance. This fact allows one to use the admittance spectroscopy for defect characterization in the space charge regions [25] and at heterointerfaces [26].

Also the helpful experimental approach is the study of heterointerface influence on the charge carrier transport by means of the dark IV characteristics' measurements in a wide temperature range and analysis of diode parameter temperature dependence.

For detailed study of III-V/Ge heterostructure, the experimental approach consisting in "subcell" fabrication is applied. The $\mathrm{p}-\mathrm{n}$ junction in the Ge substrate was formed due to the diffusion of $\mathrm{P}$ atoms from the growing GaInP nucleation layer. Such grown heterostructure completely reproduces the narrow gap Ge subcell in MJ SC. A ${ }^{++}$GaAs contact layer was grown for subsequent fabrication of the test $\mathrm{Ge}$ photovoltaic convertor. Then an additional postgrowth thermal treatment which is reproducing the growth conditions (temperature and growth time) of GaInP/GaInAs wide-bandgap tandem was carried out. The structures were annealed in the $\mathrm{AsH}_{3}$ atmosphere in order to prevent degradation of the GaAs cap layer.

Thus, the experimental simulation of the Ge subcell was performed by an additional thermal treatment of a single Ge cell. Such structures are referred to as "subcell" here.

\section{Results and Discussion}

To form the bottom Ge subcell, the epitaxial GaInP layers grown on the Ge substrate are used as the source for doping impurities (P) to form Ge p-n junction and as a wideband-gap window for the Ge subcell. On the one hand the III-V/Ge interface is a very critical issue from epitaxial viewpoint, because it is a boundary between chemically dissimilar materials. On the other hand properties of diffusive p-n junction are less controlled and require detailed study.

There is a major problem connected with the growth of defect-free III-V semiconductors on a nonsimilar (group IV) germanium substrate. By using the RAS in situ method, the most optimal conditions for the growth of perfect III-V layers on a Ge were determined. The conditions of deoxidation of Ge substrates and the suppression of autodoping by germanium of the photoactive parts of MJ SC [15] structure have been found due to single-wavelength RA signal temporal dependence measurement during the growth process.

The autodoping process has been suppressed in our experiments by growing a nonphotoactive $n-G_{0.99} \mathrm{In}_{0.01}$ As buffer at rather low growth temperature. Due to the electrooptical effect, the RAS is sensitive to the doping level (Figure 3). 


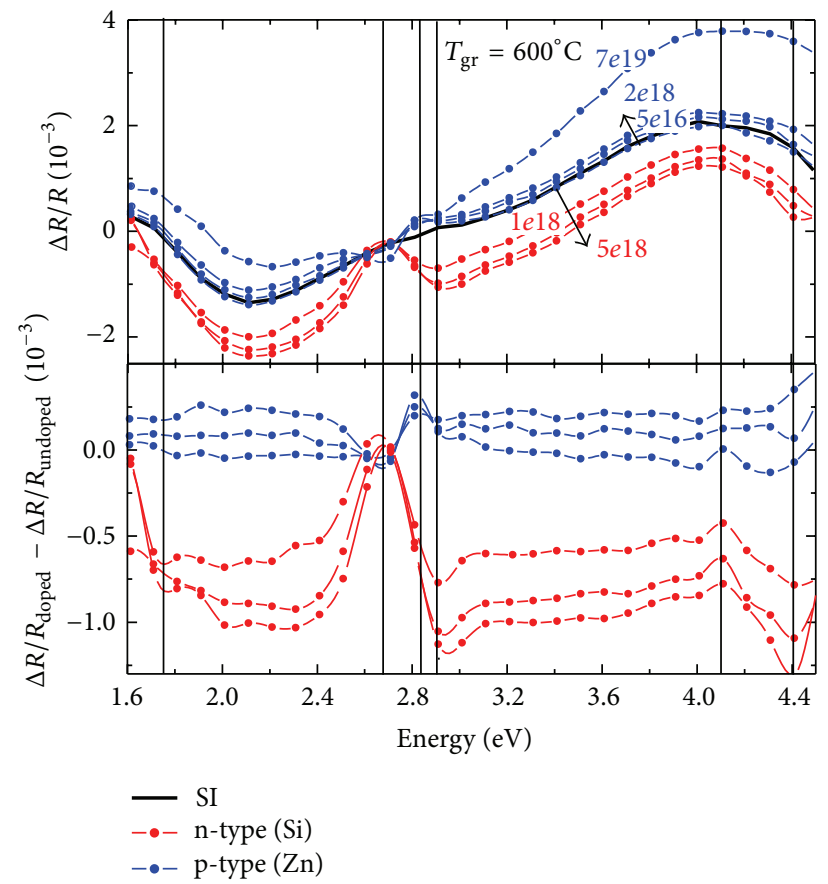

FIGURE 3: RA spectra (upper part) and $\Delta$ RAS spectra $\left(\Delta R / R_{\text {doped }}-\Delta R / R_{\text {undoped }}\right)$ for p-type: $\mathrm{Zn}$ (blue) and n-type: $\mathrm{Si}$ (red) doped $\mathrm{Ga} \mathrm{a}_{0.99} \mathrm{In}_{0.01} \mathrm{As}$ layers at $600^{\circ} \mathrm{C}$ growth temperature; energies near the critical points of dielectric function are marked by vertical black lines.

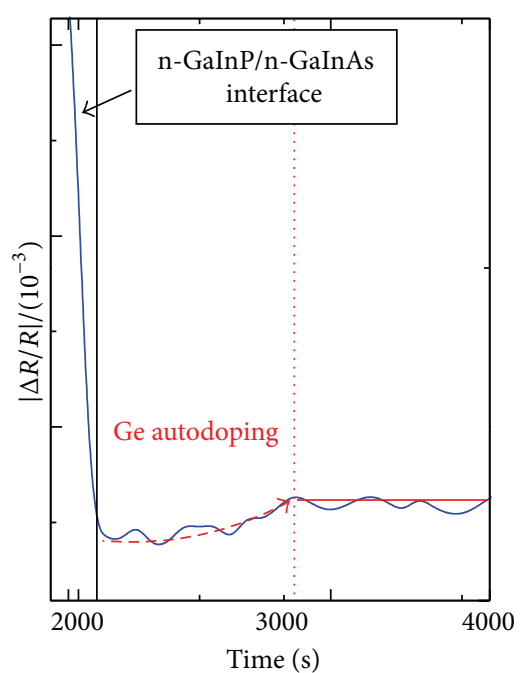

(a)

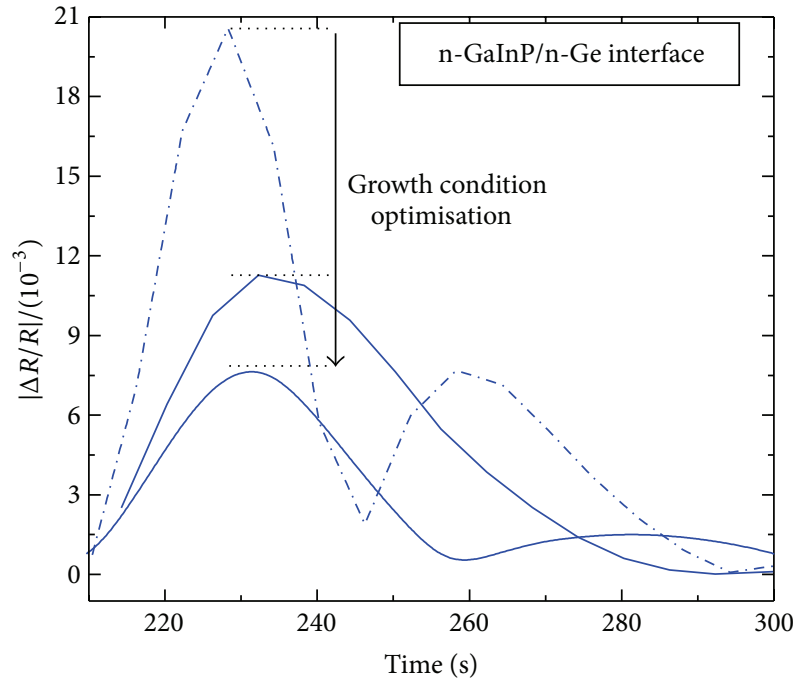

(b)

FIGURE 4: The in situ record of the single-wavelength $(3.8 \mathrm{eV})$ RA signal: (a) during GaInAs buffer growth (Ge autodoping suppression is shown) and (b) during GaInP on Ge nucleation (optimization interface quality is shown).

The red dotted line in Figure 4(a) marks the growth time (interval of about 1000 seconds) after which the doping level comes to saturation corresponding to the doping level of the $\mathrm{n}-\mathrm{Ga}_{0.99} \mathrm{In}_{0.01}$ As buffer. Taking into account the $\mathrm{Ga}_{0.99} \mathrm{In}_{0.01}$ As layer growth rate $(8.5 \AA / \mathrm{s})$ one can conclude that the thickness of the layer, when autodoping with germanium influences on RA signal level, is in the range $0.7-0.9 \mu \mathrm{m}$.
The interface quality was determined by analyzing the RA signal temporal dependence during growth. It is known that the RA signal at the interface directly correlates with a rough interface [27]. Three RA transients from GaInP layers grown on Ge after different preepitaxial treatments are shown in Figure 4(b). The maximum RA signal rise was observed at growth on a not completely deoxidized germanium substrate. 


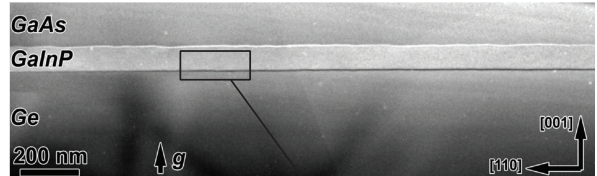

(a)

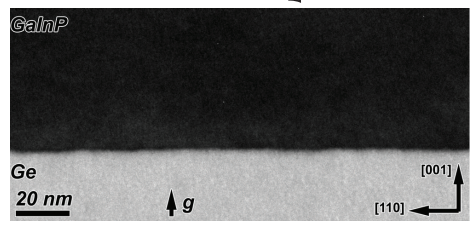

(b)

FIGURE 5: Bright field TEM image of (1-10) cross-section of a SC structure (a) and dark field TEM image of (1-10) cross-section of the GaInP/Ge interface (b). The TEM images were obtained in twobeam condition with a diffraction vector $g=(002)$.

Further growth of a bulk semiconductor structure resulted in imperfect crystallization of the epitaxial layers. This has been confirmed by comparison of the short-wavelength reflectance signal recorded in the single-wavelength mode with a successful run.

The minimum relative value of the RA signal at the $\mathrm{GaInP}_{2} / \mathrm{Ge}$ interface achieved was 8 . In this case, the reflection signal level remained unchanged during the following growth of the bulk crystal. A high structural quality of the GaInP layers and a sharpness of the GaInP/Ge interface were confirmed by TEM study (Figure 5). No dislocations or any growth related defects were observed.

Thus, the approach with in situ measurements allows determining the key parameters for successful nucleation on Ge substrates including those which are difficult to obtain by means of ex situ methods.

In spite of such quality a number of new phenomena associated with a GaInP/Ge heterostructure were found during investigation by means of a number of approaches.

In particular, it has been shown $[28,29]$ that there are excess current components, which, at reverse bias, gave the saturation current density in the range of $\left(10^{-4}-10^{-3}\right) \mathrm{A} / \mathrm{cm}^{2}$ for $\mathrm{Ge}$ p-n junction formed during the MOVPE diffusion process. Previously, it was believed that, in the Ge p-n junctions, the forward current flow mechanism is diffusion (the ideality factor $A_{d}=1$ ).

For such "subcells" (Ge subcell structure and their growth conditions were experimentally simulated as described above) the dark forward and reverse IV curves were analyzed for the temperature range of $90-330 \mathrm{~K}$. The approximation of Ge subcell IV curves by means of only one-exponential (diffusion) model in the whole temperature range was not absolutely accurate. It was shown that an excess current should be taken into account for Ge subcells and that the appropriate model for approximation of IV curves is twoexponential. As a result of the diode parameter analysis, the nature of the excess current was determined as either tunneling or thermotunneling.

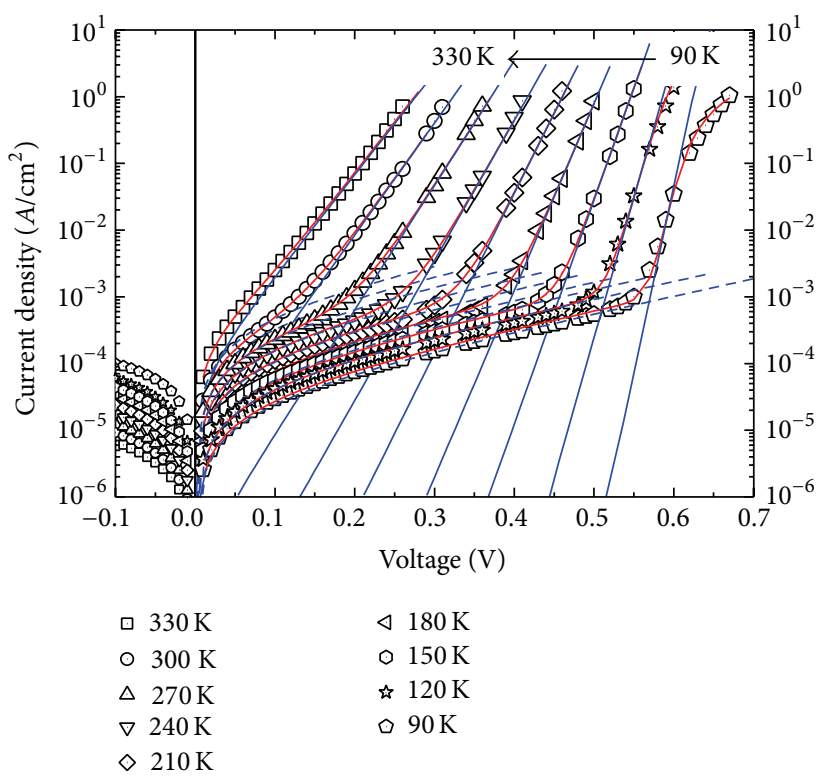

FIGURE 6: Forward and reverse dark IV characteristics of a Ge "subcell" at different temperatures (from 90 to $330 \mathrm{~K}$ ). Symbols are the experimental data: red line: the calculations by the twoexponential model, blue line: the diffusion components $\left(A_{d}=1\right)$, and blue separated line: the tunneling components $\left(E_{t}=0.17 \mathrm{~V}\right)$.

A number of samples (especially after high temperature treatment) showed "not classic" IV curves in whole temperature range (Figure 6). For these samples the approximation of the forward branch of the IV curves by the sum of the components of exponential form (2) has shown that, in the operation temperature range of 90-330 K, the forward current consists of the diffusion component and the excess component:

$J=J_{o d} \cdot\left[\exp \left(\frac{V-J \cdot R_{s}}{E_{d}}\right)-1\right]+J_{o t} \cdot\left[\exp \left(\frac{V-J \cdot R_{s}}{E_{t}}\right)\right]$,

where $J$ is the current density in the external circuit, $V$ is voltage, $R_{s}$ is the series resistance of the device, $J_{o d}$ is the saturation current of the diffusion component (preexponent), $J_{o t}$ is the preexponent ("saturation" current) of the excess (tunneling) component, $E_{t}$ is the characteristic potential of the tunneling component, and $E_{d}$ is the characteristic (thermal) potential of the diffusion component, which may be expressed through the ideality factor $A_{d}=1$ :

$$
E_{d}=\frac{k T}{q} \cdot A_{d}=\frac{k T}{q},
$$

where $q$ is the electron charge, $k$ is the Boltzmann constant, and $T$ is the absolute temperature.

The analysis of diode parameters of the second term in the expression (2) showed that the excess current is characterized by a thermally independent potential $E_{t}(0.17 \mathrm{~V})$ and weak (in comparison with the thermal activation dependence) exponential temperature dependence of $J_{o t}$. The temperature 


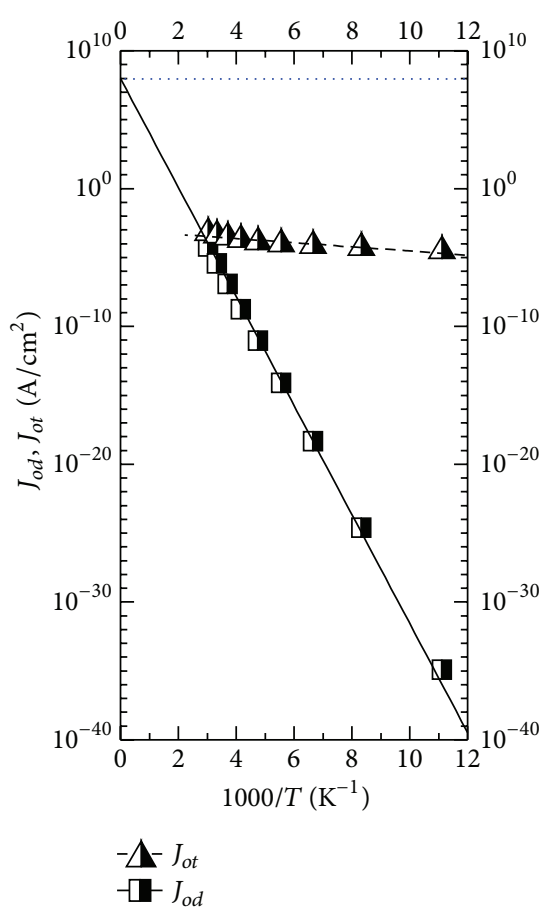

(a)

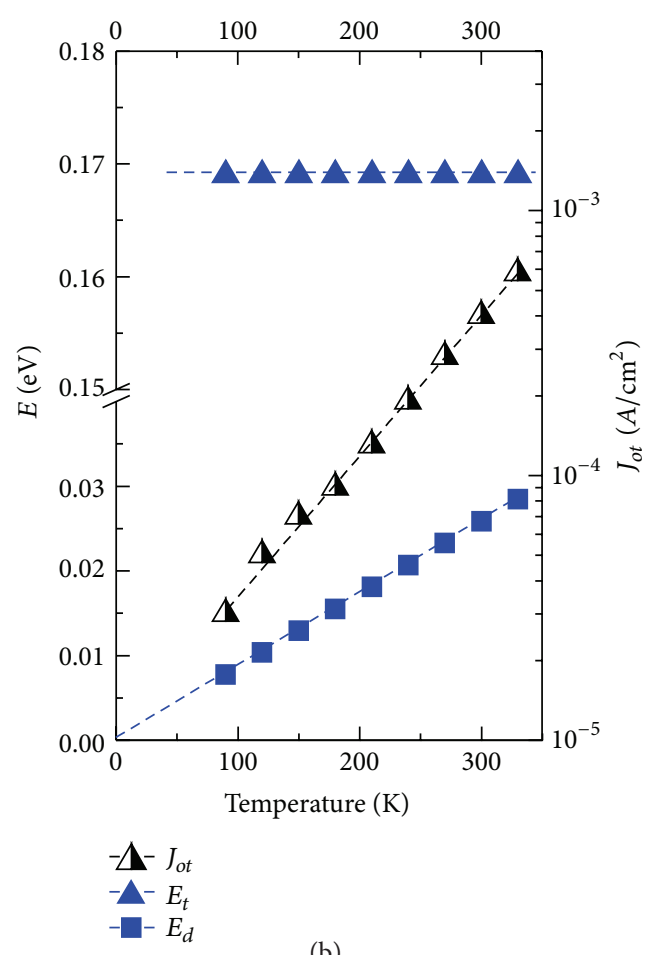

(b)

Figure 7: Dependencies of the Ge "subcell" diode parameters of diffusion $\left(E_{d}, J_{o d}\right)$ and tunneling $\left(E_{t}, J_{o t}\right)$ current components on the value of inverse (a) and forward (b) absolute temperature.

coefficient is $b=\Delta \ln J_{o t} / \Delta T=3 \div 9 \times 10^{-3} \mathrm{~K}^{-1}$ ) (Figure 7). That allows characterizing the mechanism as a tunneling current.

However, in most cases, the excess component is not observed in the dark IV characteristics of Ge subcell at RT. However, at lower temperatures (especially at $T<200 \mathrm{~K}$ ) excess current has been detected. Thus, in this case the dark IV characteristic of a Ge "subcell" can be described by twoexponential model similarly to (2):

$J=J_{o d} \cdot\left[\exp \left(\frac{V-J \cdot R_{s}}{E_{d}}\right)-1\right]+J_{o t t} \cdot\left[\exp \left(\frac{V-J \cdot R_{s}}{E_{t t}}\right)\right]$

where the second term is thermotunneling current, $J_{\text {ott }}$ is the preexponent ("saturation" current), and $E_{t t}$ is the characteristic potential of the thermotunneling component.

Temperature dependence of $J_{\text {ott }}$ (Figure 8(a)) at low temperatures is rather weak (in comparison with the thermal activation dependence) but at high temperatures can be approximated by an exponential function:

$$
J_{\text {ott }}=J_{\infty} \cdot \exp \left(\frac{-E_{g 0}}{A_{\beta} \cdot k T}\right),
$$

where $E_{g 0}=0.78 \mathrm{eV}[20]$ is the extrapolated value of the $\mathrm{Ge}$ bandgap at $T \rightarrow 0, A_{\beta}$ is the coefficient ("ideality factor") independent of temperature and approximately equal to 1.5 , and $J_{\infty}$ is the constant, which characterizes $J_{o t t}$ at $1 / T \rightarrow 0$ (experimentally determined value of $J_{\infty}$ is $5000 \mathrm{~A} / \mathrm{cm}^{2}$ ).
The characteristic potential $E_{t t}$ at high temperatures can be well approximated by a function which increases linearly with temperature (Figure 8(b)):

$$
E_{t t}=A_{\beta} \cdot \frac{k T}{q} .
$$

Thus, in this case the excess current has thermal activation dependence of $J_{o t}$ (with activation energy $E_{a}=E_{g} / A_{\beta}$ and ideality factor $\left.A_{\beta}=E /(k T / q) \sim 1.5\right)$ at high temperatures $T \gtrsim 200 \mathrm{~K}$, but the current acquires the tunneling character, when temperature is decreasing $\left(E_{t t 0}=0,016 \div\right.$ $0,019 \mathrm{~V}$ ) (Figure 8). The availability of both the tunnel and the thermoinjection parts on $J_{o t t}(T)$ dependence allows characterizing the mechanism as thermotunneling.

Although the influence of thermotunneling current on the efficiency at RT and high temperature (concentrator application) can be neglected, the two-exponential model is more accurate for description of current transport in $\mathrm{Ge}$ subcell (formed by MOCVD) into the wide temperature range.

The nature of the excess currents cannot be explained by dislocations (as it is known for heterojunctions) because of high quality of the $\mathrm{GaInP}_{2} / \mathrm{Ge}$ interface (Figure 5). A possible reason for the excess currents was investigated in detail in our paper [30].

The dark IV curves at $100 \mathrm{~K}$ for the Ge "subcells" as well as for the GaInP/GaInAs/Ge MJ SC (Figure 9) clearly demonstrate an S-shape behavior, which is somewhat similar to Shockley's dynistor. The "set-on" voltage of the S-shape 

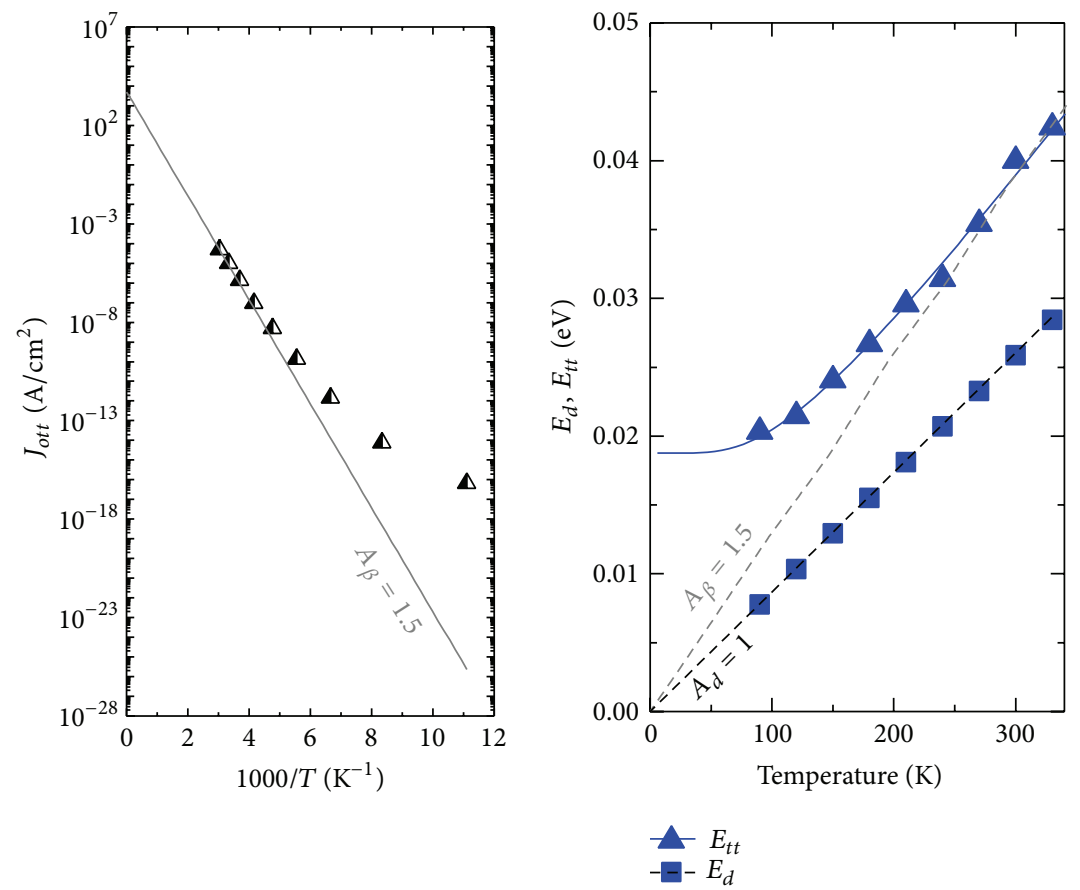

(a)

(b)

FIGURE 8: Dependencies of the Ge "subcell" diode parameters of thermotunneling $\left(E_{t t}, J_{o t t}\right)$ current component and thermal potential $\left(E_{d}\right)$ of the diffusion current on the value of inverse (a) and forward (b) absolute temperature.

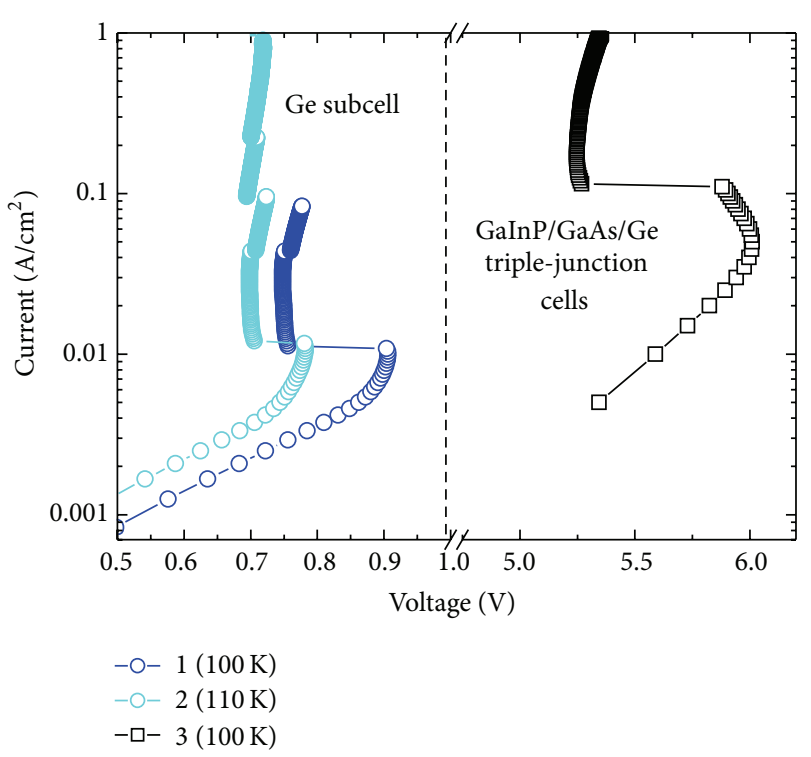

FIGURE 9: Dark IV curves recorded in a current control mode for the Ge "subcell" structure at $100 \mathrm{~K}$ (1) and $110 \mathrm{~K}$ (2), GaInP/GaAs/Ge triple-junction solar cell at $100 \mathrm{~K}(3)$.

increases with temperature decrease (from $0.78 \mathrm{~V}$ at $110 \mathrm{~K}$ to $0.9 \mathrm{~V}$ at $100 \mathrm{~K}$ for Ge "subcells").

The admittance spectroscopy was applied for study of the GaInP/Ge heterointerface. The "subcell" structure exhibits a

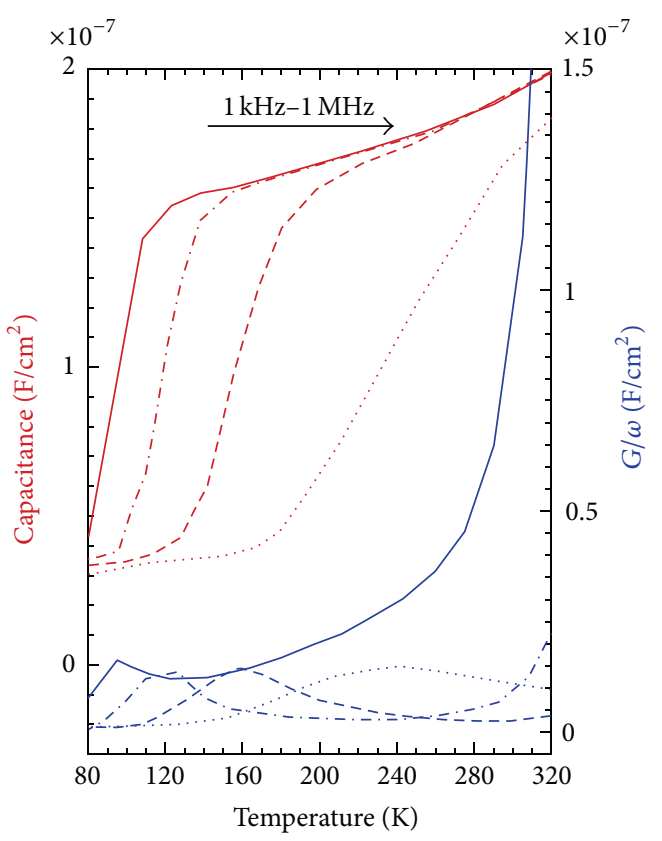

Figure 10: $C-T-\omega$ and $G / \omega-T-\omega$ curves for GaInP/Ge subcell heterostructures [20].

characteristic step in the $C(f, T)$ curves (Figure 10) accompanied by peaks in the $G(f, T)$ curves. The obtained value of the activation energy, in the range of $0.05 \sim 0.1 \mathrm{eV}$, corresponds to the effective height of the potential barrier. 
The experimental IV curves and the admittance spectroscopy data indicate [30] the presence of the undesirable potential barrier for majority of charge carriers in "subcell" heterostructures. The origin of this barrier formation could be related to the diffusion process at the GaInP/Ge interface. In fact, simultaneously with phosphorous ( $\mathrm{V}$ group atom) diffusion from GaInP to the Ge wafer, which forms the $\mathrm{n}$-type region of the $\mathrm{p}-\mathrm{n}$ junction in $\mathrm{p}-\mathrm{Ge}$, gallium and indium diffusion to $\mathrm{Ge}$ also exists. As $\mathrm{Ga}$ and In are acceptor impurities for $\mathrm{Ge}$, they could form a p-type region. The diffusion coefficient of phosphorous $\left(\sim 2.4 \times 10^{-11} \mathrm{~cm}^{2} / \mathrm{s}\right)$ at growth temperature is much higher compared to that of both gallium $\left(\sim 3.5 \times 10^{-13} \mathrm{~cm}^{2} / \mathrm{s}\right)$ and indium $\left(\sim 5.9 \times 10^{-13} \mathrm{~cm}^{2} / \mathrm{s}\right)$ [31]. That causes deeper diffusion of phosphorous into the Ge wafer. However, the solubility limit of Ga in Ge $(4.5 \times$ $10^{20} \mathrm{~cm}^{-3}$ ) is significantly higher than that of phosphorous $\left(5 \times 10^{19}\right) \mathrm{cm}^{-3}[32,33]$. Therefore a local concentration of $\mathrm{Ga}$ atoms diffused to $\mathrm{Ge}$ at the $\mathrm{GaInP} / \mathrm{Ge}$ interface could be significantly higher compared to that of $\mathrm{P}$ atoms, which leads to a narrow region with a p-type doping domination. The inversion of the conduction type in this region near the interface causes the potential barrier for electrons, which can affect the charge carrier transport properties of a Ge solar cell.

The schematic band diagram with the potential barrier at the $\mathrm{n}-\mathrm{GaInP} / \mathrm{n}-\mathrm{Ge}$ interface was proposed for the "subcell" structures (Figure 11). The behavior of the experimental IV curves and admittance spectra was reproduced by the numerical simulations using the proposed model with a $60 \mathrm{~nm}$ thick p-type Ge $\left(3 \times 10^{17} \mathrm{~cm}^{-3}\right)$ layer introduced at the interface between the (n)GaInP and (n)Ge, which forms the potential barrier of about $0.1 \mathrm{eV}$.

The dark IV curves at $100 \mathrm{~K}$ and $300 \mathrm{~K}$ for the heterostructures with ("subcell") and without potential barrier at the $\mathrm{GaInP} / \mathrm{Ge}$ interface were simulated (Figure 12) using the AFORS-HET software [34].

The simulations have shown a good agreement with experimental data meaning that the presence of the potential barrier for electrons at the $\mathrm{GaInP} / \mathrm{Ge}$ interface can explain the observed behavior at low temperatures for the "subcell" structures.

The undesirable potential barrier for majority of charge carriers at the $n-G a I n P / n-G e$ heterointerface seems to be a general problem of the III-V/IV heteroepitaxial interfaces.

\section{Summary}

The review of important but little-investigated problems connected with III-V/Ge heterostructure in the GaInP/ GaInAs/Ge multijunction solar cells grown by MOVPE is presented. Experimental in situ and ex situ investigation approaches are analyzed.

A combination of reflectance and reflectance anisotropy spectroscopy in situ methods has been applied for investigating III-V semiconductor growth on Ge substrates. By means of the in situ methods, the conditions for highquality material growth on Ge substrates have been found. The growth parameters of III-V buffer layer $\left(\mathrm{Ga}_{0.99} \mathrm{In}_{0.01} \mathrm{As}\right)$

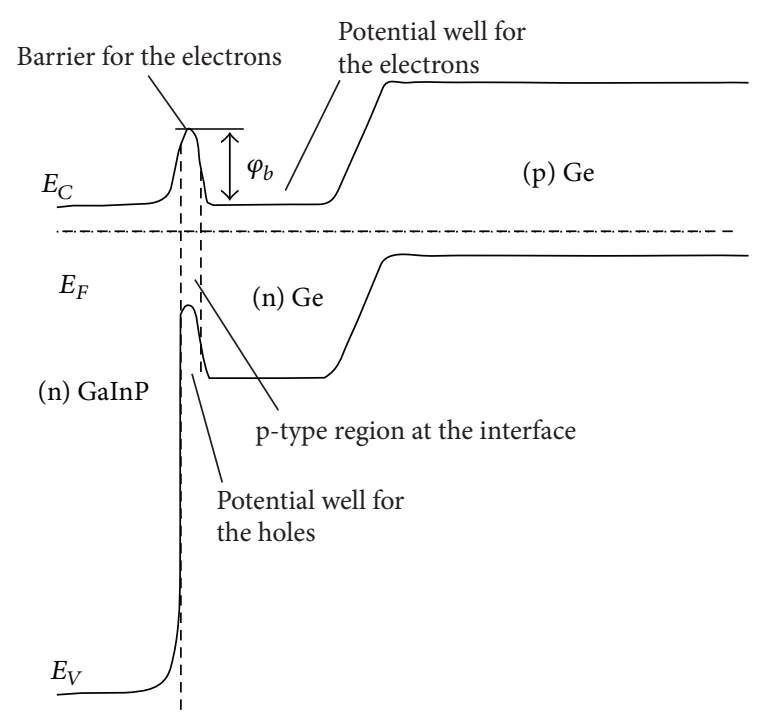

FIGURE 11: Schematic band diagram at equilibrium proposed for $\mathrm{Ge}$ "subcell" structures.

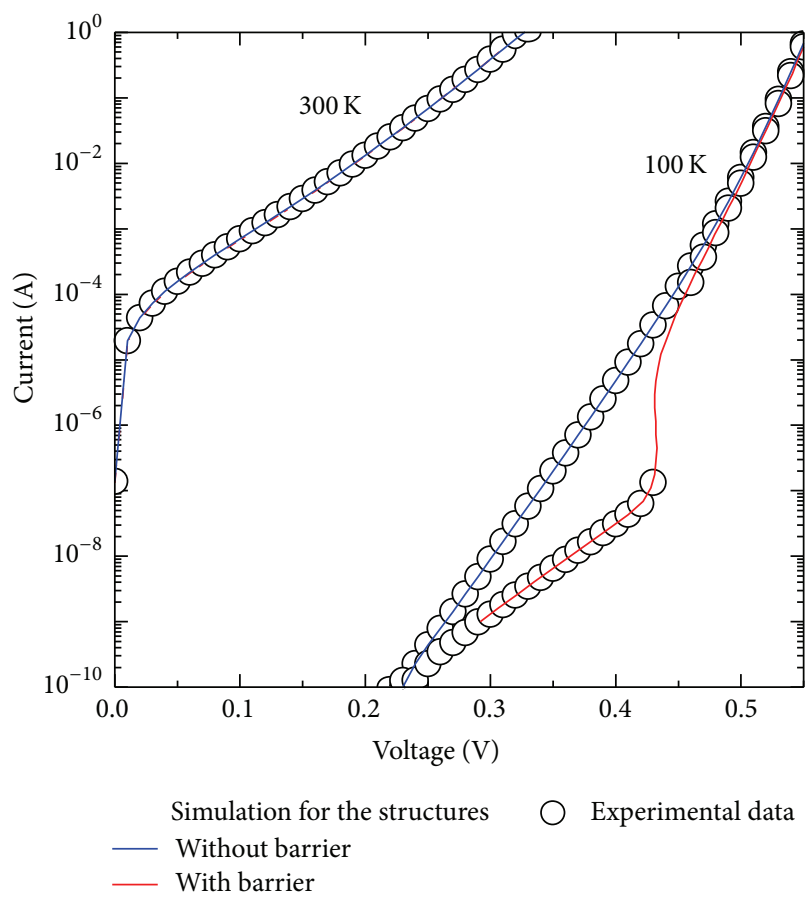

FIGURE 12: The simulated IV curves for the heterostructures with and without a potential barrier at the GaInP/Ge interface at 300 and $100 \mathrm{~K}$.

for preventing autodoping by Ge of the photoactive MJ SC structure have been established.

Photovoltaic properties of n-GaInP/n-p Ge heterostructure, which is a narrow-band subcell of the triple-junction GaInP/GaInAs/Ge solar cell, have been investigated ex situ. It has been shown (from the temperature dependence of the dark IV curves, 77-330 K) that there is excess current (beside the diffusion one) in the Ge photovoltaic p-n junctions. The excess current has the tunneling or thermotunneling 
character. The values of the diode parameters for both current flow mechanisms have been determined.

The presence of the potential barrier at the $n-G a I n P / n-G e$ interface has been confirmed by the admittance spectroscopy, low-temperature dark IV curve measurements, and numerical simulations. The origin of this barrier formation could be related to the parasitic diffusion of III group atoms into Ge substrate during heterostructure growth.

\section{Conflict of Interests}

The authors declare that there is no conflict of interests regarding the publication of this paper.

\section{Acknowledgments}

The authors want to acknowledge that this work has been supported by the Contract no. 14.B25.31.0020 from the Russian Ministry of Education and Science (Resolution no. 220). The authors thank A. A. Usikova and A. V. Malevskaya for preparation of the samples by photolithography.

\section{References}

[1] M. A. Green, K. Emery, Y. Hishikawa, W. Warta, and E. D. Dunlop, "Solar cell efficiency tables (version 41)," Progress in Photovoltaics: Research and Applications, vol. 21, no. 1, pp. 1-11, 2013.

[2] R. R. King, D. Bhusari, D. Larrabee et al., "Solar cell generations over $40 \%$ efficiency," Progress in Photovoltaics: Research and Applications, vol. 20, no. 6, pp. 801-815, 2012.

[3] M. Wiemer, V. Sabnis, and H. Yuen, " $43.5 \%$ efficient lattice matched solar cells," in High and Low Concentrator Systems for Solar Electric Applications VI, vol. 810804 of Proceedings of SPIE, San Diego, Calif, USA, September 2011.

[4] R. R. King, D. C. Law, K. M. Edmondson et al., " $40 \%$ efficient metamorphic GaInP/GaInAs/Ge multijunction solar cells," Applied Physics Letters, vol. 90, no. 18, Article ID 183516, 2007.

[5] D. E. Aspnes, J. P. Harbison, A. A. Studna, and L. J. Flores, "Application of reflectance difference spectroscopy to molecular-beam epitaxy growth of GaAs and AlAs," Journal of Vacuum Science \& Technology A, vol. 6, no. 3, pp. 1327-1332, 1988.

[6] J.-T. Zettler, M. Pristovsek, T. Trepk et al., "Response of the surface dielectric function to dynamic surface modifications: application of reflectance anisotropy spectroscopy and spectroscopic ellipsometry," Thin Solid Films, vol. 313-314, pp. 537-543, 1998.

[7] P. Kurpas, M. Arens, D. Gutsche, E. Richter, and M. Weyers, "Growth monitoring of GalnP/GaAs heterojunction bipolar transistors by reflectance anisotropy spectroscopy," Journal of Crystal Growth, vol. 195, no. 1-4, pp. 217-222, 1998.

[8] G. B. Stringfellow, Organometallic Vapor-Phase Epitaxy: Theory and Practice, Academic Press, San Diego, Calif, USA, 2nd edition, 1999.

[9] D. E. Aspnes and A. A. Studna, "Anisotropies in the above-bandgap opticalspectra of cubic semiconductors," Physical Review Letters, vol. 54, pp. 1956-1959, 1985.
[10] V. L. Berkovits, L.F. Ivantsov, V. a. Kiselev et al., "Polarization spectra of optical transitions at a clean GaAs (110) surface," Journal of Experimental and Theoretical Physics Letters, vol. 41, p. 551, 1985.

[11] V. L. Berkovits, V. N. Bessolov, T. N. Lvova, V. I. Safarov, R. V. Khasieva, and B. V. Tsarenkov, "Fermi-level movement at $\mathrm{GaAs}(001)$ surfaces passivated with sodium sulfide solutions," Journal of Applied Physics, vol. 70, no. 6, pp. 3707-3711, 1991.

[12] H. Tanaka, E. Colas, I. Kamiya, D. E. Aspnes, and R. Bhat, "In situ determination of free-carrier concentrations by reflectance difference spectroscopy," Applied Physics Letters, vol. 59, no. 26, pp. 3443-3445, 1991.

[13] P. Wolfram, E. Steimetz, W. Ebert, B. Henninger, and J.-T. Zettler, "Growth of InGaAsP/InP-laser structures monitored by using RAS techniques," Journal of Crystal Growth, vol. 248, pp. 240-243, 2003.

[14] K. Haberland, M. Zorn, A. Klein et al., "In-situ determination of interface roughness in MOVPE-grown visible VCSELs by reflectance spectroscopy," Journal of Crystal Growth, vol. 248, pp. 194-200, 2003.

[15] N. A. Kalyuzhnyy, V. M. Lantratov, S. A. Mintairov et al., "Insitu monitoring during mocvd growth of the triple-junction $\mathrm{GaInP} / \mathrm{Ga}(\mathrm{In}) \mathrm{As} / \mathrm{Ge}$ solar cells," in Proceedings of the 23th European Photovoltaic Solar Energy Conference (EPSEC '08), pp. 803-810, Valencia, Spain, September 2008.

[16] N. A. Kalyuzhnyy, S. A. Mintairov, M. A. Mintairov, and V. M. Lantratov, "Investigation of photovoltaic devices crystallization in MOCVD with in-situ monitoring," in Proceedings of the 24th European Photovoltaic Solar Energy Conference (EPSEC '09), pp. 538-544, Hamburg, Germany, September 2009.

[17] V. M. Andreev, N. A. Kalyuzhnyy, V. M. Lantratov, S. A. Mintairov, M. Z. Shvarts, and N. K. Timoshina, "Concentrator GaInP/GaAs tandem solar cells with in-situ monitoring of the MOCVD growth," in Proceedings of the 22nd European Photovoltaic Solar Energy Conference (EPSEC '07), pp. 542-547, Milan, Italy, September 2007.

[18] A. Zunger and S. Mahajan, in Handbook onSemiconductors, S. Mahajan, Ed., vol. 3, p. 1399, Elsevier, Amsterdam, The Netherlands, 2nd edition, 1994.

[19] V. M. Lantratov, N. A. Kalyuzhnyǐ, S. A. Mintairov, N. K. Timoshina, M. Z. Shvarts, and V. M. Andreev, "High-efficiency dual-junction GaInP/GaAs tandem solar cells obtained by the method of MOCVD," Semiconductors, vol. 41, no. 6, pp. 727-731, 2007.

[20] R. A. Smith, Semiconductors, Cambridge University Press, 1959.

[21] X. He and M. Razeghi, "Investigation of the heteroepitaxial interfaces in the GaInP/GaAs superlattices by high-resolution $\mathrm{x}$-ray diffractions and dynamical simulations," Journal of Applied Physics, vol. 73, no. 7, pp. 3284-3290, 1993.

[22] J. M. Olson, R. K. Ahrenkiel, D. J. Dunlavy, B. Keyes, and A. E. Kibbler, "Ultralow recombination velocity at $\mathrm{Ga} 0.5 \mathrm{In} 0.5 \mathrm{P} / \mathrm{GaAs}$ heterointerfaces," Applied Physics Letters, vol. 55, no. 12, pp. 1208-1210, 1989.

[23] M. O. Watanabe and Y. Ohba, "Interface properties for $\mathrm{GaAs} / \mathrm{InGaAlP}$ heterojunctions by the capacitance-voltage profiling technique," Applied Physics Letters, vol. 50, no. 14, pp. 906908, 1987.

[24] A. S. Gudovskikh, R. Chouffot, J. P. Kleider et al., "New method for interface characterization in heterojunction solar cells based on diffusion capacitance measurements," Thin Solid Films, vol. 516, no. 20, pp. 6786-6790, 2008. 
[25] D. V. Lang, J. D. Cohen, and J. P. Harbison, "Measurement of the density of gap states in hydrogenated amorphous silicon by space charge spectroscopy," Physical Review B, vol. 25, no. 8, pp. 5285-5320, 1982.

[26] A. S. Gudovskikh, J.-P. Kleider, J. Damon-Lacoste et al., "Interface properties of a-Si:H/c-Si heterojunction solar cells from admittance spectroscopy," Thin Solid Films, vol. 511-512, pp. 385389, 2006.

[27] M. Zorn and M. Weyers, "Application of reflectance anisotropy spectroscopy to laser diode growth in MOVPE," Journal of Crystal Growth, vol. 276, no. 1-2, pp. 29-36, 2005.

[28] N. A. Kalyuzhnyy, A. S. Gudovskikh, V. V. Evstropov et al., "Current flow and efficiency of Ge p-n junctions in triplejunction $\mathrm{GaInP} / \mathrm{Ga}(\mathrm{In}) \mathrm{As} / \mathrm{Ge}$ solar cells for space applications," in Proceedings of the 25th European Photovoltaic Solar Energy Conference and Exhibition and 5th World Conference on Photovoltaic Energy Conversion (EU PVCEC-WCPEC '10), pp. 865871, Valencia, Spain, September 2010.

[29] N. A. Kalyuzhnyy, A. S. Gudovskikh, V. V. Evstropov et al., "Germanium subcells for multijunction GaInP/GaInAs/Ge solar cells," Semiconductors, vol. 44, no. 11, pp. 1520-1528, 2010.

[30] A. S. Gudovskikh, K. S. Zelentsov, N. A. Kalyuzhnyy, V. V. Evstropov, V. M. Lantratov, and S. A. Mintairov, "Interface properties of GaInP/Ge hetero-structure sub-cells of multijunction solar cells," Journal of Physics D: Applied Physics, vol. 45, no. 49, Article ID 495305, 2012.

[31] N. B. Hannay, Semiconductors, Reinhold, New York, NY, USA, 1959.

[32] N. D. Zakharov, V. N. Rozhanskij, and R. L. Korchazhkina, "Defects evolving from the decomposition of a solid solution ofphosphorus in germanium," Fizika Tverdogo Tela, vol. 16, pp. 1444-1450, 1974 (Russian).

[33] V. I. Fistul, A. G. Yakovenko, A. A. Gvelesiani, V. N. Tsygankov, and R. L. Korchazhkina, "Solubility and precipitation of electrically active P in Ge," Inorganic Materials, vol. 11, p. 457, 1975 (Russian).

[34] R. Stangl, M. Kriegel, and M. Schmidt, "AFORS-HET, version 2.2, a numerical computer program for simulation of heterojunction solar cells and measurements," in Proceedings of the 4th IEEE World Conference on Photovoltaic Energy Conversion (WCPEC-4 '06), pp. 1350-1353, Waikoloa, Hawaii, USA, May 2006. 

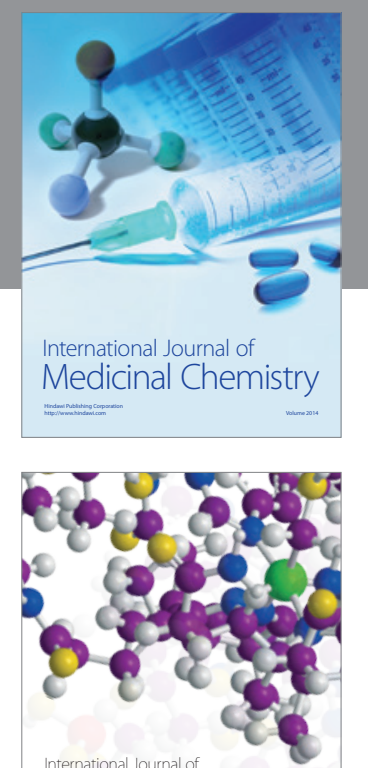

\section{Carbohydrate} Chemistry

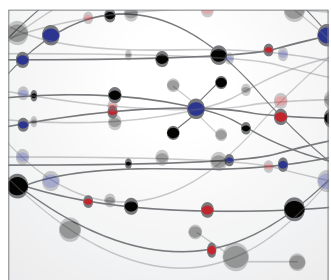

The Scientific World Journal
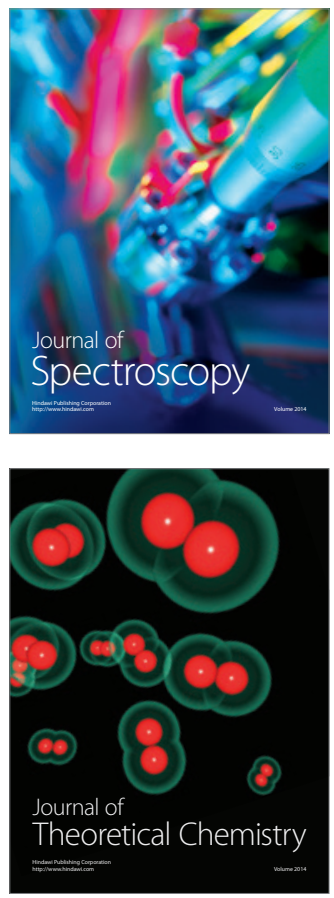
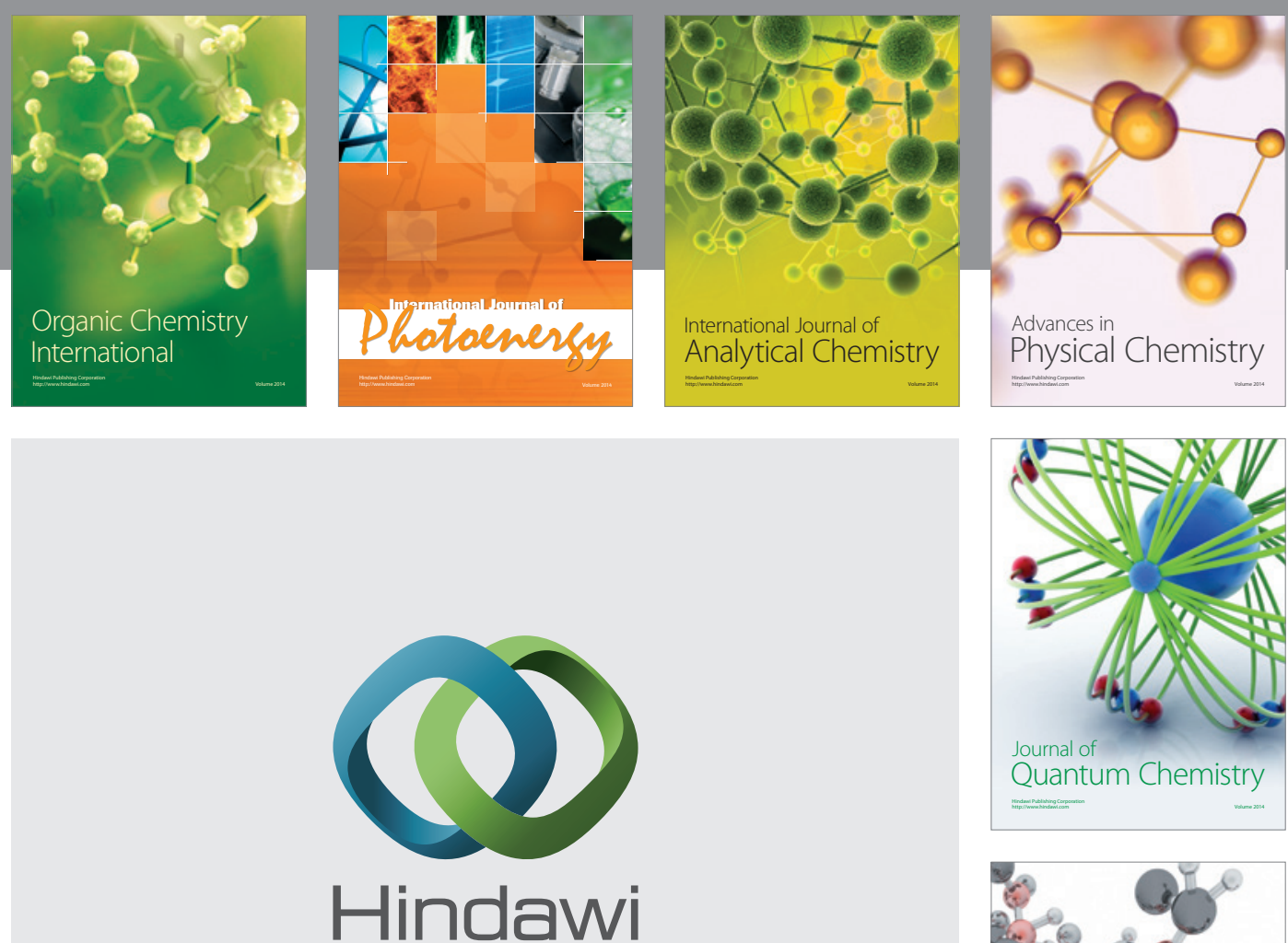

Submit your manuscripts at

http://www.hindawi.com

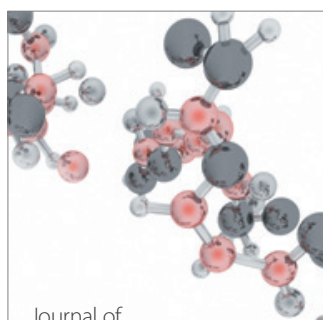

Analytical Methods

in Chemistry

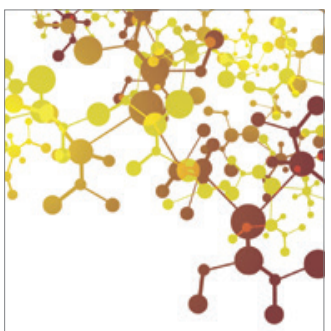

Journal of

Applied Chemistry

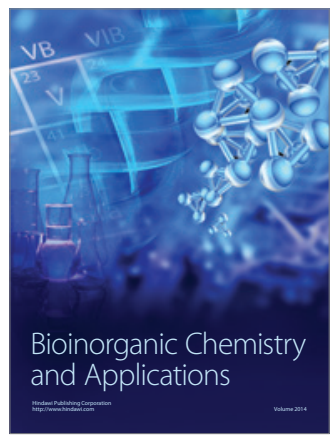

Inorganic Chemistry
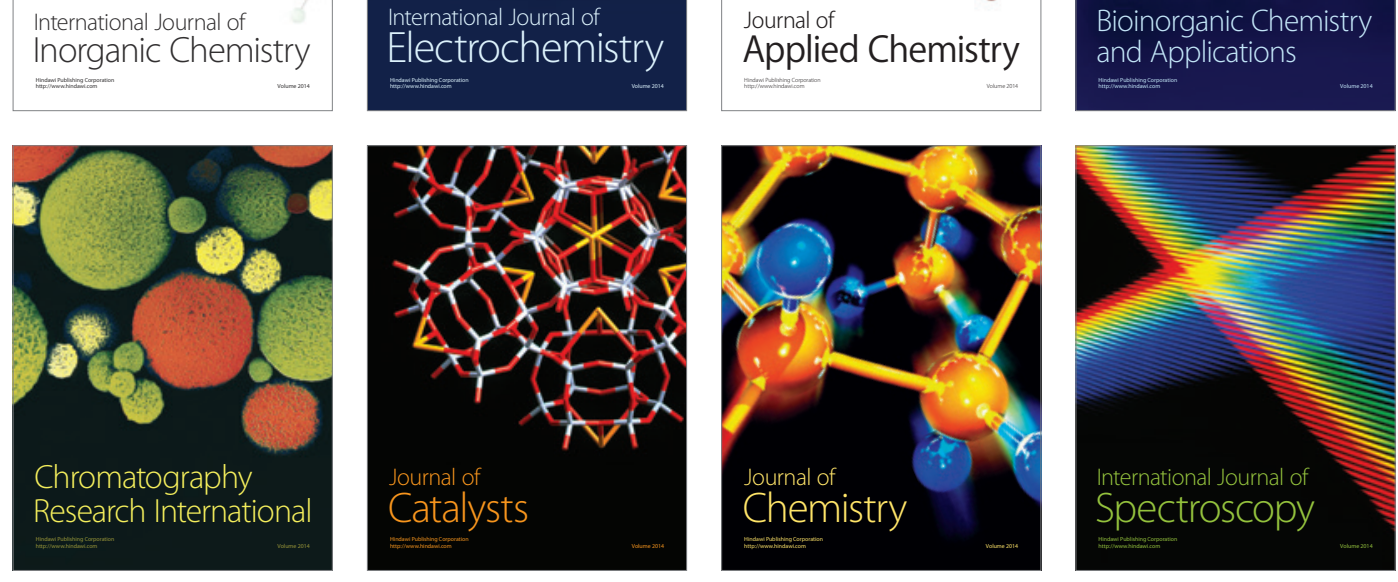